\title{
Numerical modelling of axisymmetric electromagnetically driven flows in thin layers
}

\author{
Sergey A. Suslov ${ }^{1} \quad$ Sergio Cuevas ${ }^{2}$
}

(Received 12 December 2016; revised 18 August 2017)

\begin{abstract}
We present the results of the numerical modelling of deceptively simple steady axisymmetric electromagnetically driven flows in thin disk-like layers of a weakly conducting electrolyte. The fluid motion is caused by an azimuthally acting Lorentz force appearing when a radial current flows in the electrolyte layer placed on top of a magnet with a vertical polarisation. The small layer thickness and the circumferential direction of the driving force suggest that the flow in such a system should be essentially uni-directional. However, it was found that not only is the flow fully three-dimensional, but multiple solutions can exist for the same set of governing parameters.
\end{abstract}

DOI:10.21914/anziamj.v58i0.11602, (c) Austral. Mathematical Soc. 2017. Published 2017-09-05, as part of the Proceedings of the 18th Biennial Computational Techniques and Applications Conference. ISSN 1445-8810. (Print two pages per sheet of paper.) Copies of this article must not be made otherwise available on the internet; instead link directly to the DOI for this article. Record comments on this article via http://journal . austms . org. au/ojs/index.php/ANZIAMJ/comment/add/11602/0 


\section{Contents}

1 Introduction

C47

2 Problem formulation and approximation

$\mathrm{C} 48$

3 Numerical results

C51

References

C55

\section{Introduction}

Electrolyte flows driven by the Lorentz force in thin cylindrical layers [1] are often used for laboratory modelling of large atmospheric events such as hurricanes and tropical cyclones [3]. The main reason for this is that these natural phenomena, which occur on a lateral scale of hundreds of kilometres and vertical extent of 10 kilometres or less, are very thin. Another reason for using electrolytes for simulating atmospheric events is that their motion can be induced and maintained without a mechanical interference that would destroy the analogy with hurricanes. The basic physical principle here is that a fluid conducting electric current experiences the Lorentz force when placed in an external magnetic field. Specifically, when electric current flows radially and the magnetic field is predominantly vertical the resultant azimuthal Lorentz force drives axisymmetric fluid flow. There exists an extensive body of literature documenting experimental studies of such physical hurricane models (see Dolzhanskii et al. [2] and references therein). In this short paper we report results of a numerical simulation of such axisymmetric flows that reveal their surprising complexity leading to a range of instabilities experimentally observed by Pérez-Barrera et al. [6]. 


\section{Problem formulation and approximation}

Consider an electrolyte layer of depth $\mathrm{h}$ over a nonconducting solid bottom with a free top surface. The layer is contained between two vertical co-axial cylindrical electrodes with radii $R_{1}$ and $R_{2}$ and placed above a permanent disk magnet that creates a predominantly vertical magnetic field with the characteristic induction $B_{0}$. When the electric potential difference $\Delta \phi_{0}$ is applied between the electrodes the total current $J_{0} \approx 2 \pi \sigma_{e} h \Delta \phi_{0} / \ln \left(R_{2} / R_{1}\right)$, where $\sigma_{e}$ is the conductivity of electrolyte, flows radially through the layer. This current creates the Lorentz force $\mathbf{F}_{\mathrm{L}}=\mathbf{j} \times \mathbf{B}$, where $\mathbf{j}$ and $\mathbf{B}$ are the local current density and the induction of the magnetic field, respectively.

Upon adopting the magneto-static approximation of Maxwell's equations, which is valid when the electrolyte velocity and conductivity are small, the steady axisymmetric nondimensional form of Poisson's equation for the electric potential, and the momentum and continuity equations for an incompressible fluid written in cylindrical coordinates $(r, \theta, z)$ become

$$
\begin{aligned}
& \frac{\partial^{2} \phi}{\partial z^{2}}+\epsilon^{2}\left(\frac{\partial^{2} \phi}{\partial r^{2}}+\frac{1}{r} \frac{\partial \phi}{\partial r}\right)=\epsilon^{2} \mathrm{Ha}^{2}\left[B_{z}\left(\frac{\partial u_{\theta}}{\partial r}+\frac{u_{\theta}}{r}\right)-B_{r} \frac{\partial u_{\theta}}{\partial z}\right] \\
& u_{r} \frac{\partial u_{r}}{\partial r}-\frac{u_{\theta}^{2}}{r}+u_{z} \frac{\partial u_{r}}{\partial z} \\
& \quad=-\frac{1}{\operatorname{Fr}^{2}} \frac{\partial p}{\partial r}+\frac{j_{\theta} B_{z}}{\epsilon^{2} \operatorname{Re}}+\frac{1}{\operatorname{Re}}\left(\frac{\partial^{2} u_{r}}{\partial r^{2}}+\frac{1}{r} \frac{\partial u_{r}}{\partial r}-\frac{u_{r}}{r^{2}}+\frac{1}{\epsilon^{2}} \frac{\partial^{2} u_{r}}{\partial z^{2}}\right) \\
& u_{r} \frac{\partial u_{\theta}}{\partial r}-\frac{u_{r} u_{\theta}}{r}+u_{z} \frac{\partial u_{\theta}}{\partial z} \\
& \quad=\frac{1}{\epsilon^{2} \operatorname{Re}}\left(j_{z} B_{r}-j_{r} B_{z}\right)+\frac{1}{\operatorname{Re}}\left(\frac{\partial^{2} u_{\theta}}{\partial r^{2}}+\frac{1}{r} \frac{\partial u_{\theta}}{\partial r}-\frac{u_{\theta}}{r^{2}}+\frac{1}{\epsilon^{2}} \frac{\partial^{2} u_{\theta}}{\partial z^{2}}\right) \\
& u_{r} \frac{\partial u_{z}}{\partial r}+u_{z} \frac{\partial u_{z}}{\partial z}-\frac{j_{\theta} B_{r}}{\epsilon^{2} R e} \\
& \quad=-\frac{1}{\operatorname{Fr}^{2} \epsilon^{2}} \frac{\partial p}{\partial z}+\frac{1}{\operatorname{Re}}\left(\frac{\partial^{2} u_{z}}{\partial r^{2}}+\frac{1}{r} \frac{\partial u_{z}}{\partial r}+\frac{1}{\epsilon^{2}} \frac{\partial^{2} u_{z}}{\partial z^{2}}\right)
\end{aligned}
$$




$$
\frac{\partial u_{r}}{\partial r}+\frac{u_{r}}{r}+\frac{\partial u_{z}}{\partial z}=0
$$

where $\phi$ is the electric potential, $\left(\mathfrak{u}_{r}, \mathfrak{u}_{\theta}, \mathfrak{u}_{z}\right)$ are the velocities and $\left(B_{r}, 0, B_{z}\right)$ are the components of the (known) magnetic field created by a disk magnet in the radial, azimuthal and vertical directions, respectively, and $p$ is the pressure including the hydrostatic component. The electric current density components are

$$
\left(\mathfrak{j}_{r}, j_{\theta}, j_{z}\right)=\left(-\frac{\partial \phi}{\partial r}+H a^{2} u_{\theta} B_{z}, H a^{2}\left(\epsilon^{2} u_{z} B_{r}-u_{r} B_{z}\right),-\frac{\partial \phi}{\partial z}-\epsilon^{2} H a^{2} u_{\theta} B_{r}\right)
$$

Here the aspect ratio of the electrolyte layer $\epsilon$, the square of the Hartmann number $\mathrm{Ha}^{2}$, characterising the ratio of electromagnetic to viscous forces, the square of the Froude number $\mathrm{Fr}^{2}$, which is the ratio of inertial to gravitational forces, and the Reynolds number Re, quantifying the ratio of inertial to viscous forces, are

$$
\epsilon=\frac{h}{R_{2}-R_{1}}, \quad \mathrm{Ha}^{2}=\frac{\sigma_{e} B_{0}^{2} h^{2}}{4 \mu}, \quad \mathrm{Fr}^{2}=\frac{2 \mathrm{U}_{0}^{2}}{g h}, \quad \mathrm{Re}=\frac{\rho \mathrm{U}_{0}\left(\mathrm{R}_{2}-\mathrm{R}_{1}\right)}{2 \mu},
$$

where $\mathrm{g}$ is the magnitude of gravity and the velocity scale is

$$
\mathrm{U}_{0}=\frac{\sigma_{e} \Delta \phi_{0} \mathrm{~B}_{0} \mathrm{~h}^{2}}{2 \mu\left(\mathrm{R}_{2}-\mathrm{R}_{1}\right)}
$$

Introducing a geometric parameter $\alpha=\left(R_{2}+R_{1}\right) /\left(R_{2}-R_{1}\right)$, we write the boundary conditions as

$$
\begin{aligned}
& u_{r}=u_{\theta}=u_{z}=0 \text { at } z=-1, r=\alpha \pm 1 \\
& u_{z}=\frac{\partial u_{r}}{\partial z}=\frac{\partial u_{\theta}}{\partial z}=0 \text { at } z=1, \\
& \phi=0 \text { at } r=\alpha-1, \quad \phi=1 \text { at } r=\alpha+1 \\
& \frac{\partial \phi}{\partial z}=0 \text { at } z=-1, \quad \frac{\partial \phi}{\partial z}=-\epsilon^{2} \mathrm{Ha}^{2} u_{\theta} B_{r} \text { at } z=1
\end{aligned}
$$


The governing equations are solved using the multi-dimensional version of the Chebyshev pseudospectral collocation method initially proposed by $\mathrm{Ku}$ and Hatziavramidis [4, 5] and implemented by Suslov and Paolucci [7, 8]. In this method the solution is represented by a linear combination of Chebyshev polynomials of degree ranging from 0 to the pre-set values $\mathrm{N}_{\mathrm{r}}$ and $\mathrm{N}_{z}$ that define the total number of Chebyshev modes used for approximating the solutions in the radial and depth directions, respectively. The physical nondimensional domain $(r, z) \in[\alpha-1, \alpha+1] \times[-1,1]$ is first transformed into the computational domain $(x, z) \in[-1,1] \times[-1,1]$ by a simple translation $x=$ $r-\alpha$. Subsequently, a rectangular Gauss-Lobatto collocation point grid $\left[x_{k}\right] \times$ $\left[z_{l}\right]$ is introduced, where $x_{k}=\cos \left[\pi(k-1) /\left(N_{r}-1\right)\right], k=1,2, \ldots, N_{r}$ and $z_{l}=$ $\cos \left[\pi(l-1) /\left(N_{z}-1\right)\right], l=1,2, \ldots, N_{z}$. The required solution, say, $f(x, z)$ is then evaluated at these nodes. As shown by Ku and Hatziavramidis $[4,5]$ these values uniquely define the coefficients in the linear combinations of Chebyshev polynomials used in the spectral approximation of the solution. Therefore, the function values at any point within a computational domain can be found since the explicit expressions for Chebyshev polynomials are readily available. Thus the spectral accuracy of the method is ensured. At the same time as discussed by $\mathrm{Ku}$ and Hatziavramidis $[4,5]$ the collocation formulation enables one to reduce the integration and differentiation procedures to a simple matrixvector multiplication. Namely, introducing the vector $\mathbf{f}=\left[\mathrm{f}_{1}, \mathrm{f}_{2}, \ldots, \mathrm{f}_{\mathrm{N}_{\mathrm{r}}}\right]^{\mathrm{T}}$ of function values at points $x_{l}$ for a fixed value of $z$ on a rectangular grid one can write

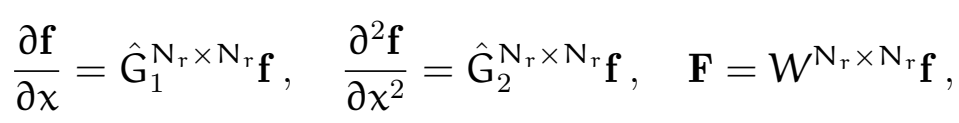

where $F=\int_{-1}^{x} f(\tilde{x}) d \tilde{x}, \hat{G}_{2}^{N_{r} \times N_{r}}=\hat{G}_{1}^{N_{r} \times N_{r}} \times \hat{G}_{1}^{N_{r} \times N_{r}}$, and the standard $N_{r} \times$ $\mathrm{N}_{\mathrm{r}}$ matrices $\hat{\mathrm{G}}_{1}^{\mathrm{N}_{\mathrm{r}} \times \mathrm{N}_{\mathrm{r}}}$ and $W^{\mathrm{N}_{r} \times \mathrm{N}_{\mathrm{r}}}$ are defined by Ku and Hatziavramidis [4, 5]. Differentiation and integration in $z$ is performed using the similar $N_{z} \times N_{z}$ matrices. For coding purposes, however, we rearrange a two-dimensional $\mathrm{N}_{\mathrm{r}} \times \mathrm{N}_{z}$ array of points into a vector of length $\mathrm{N}=\mathrm{N}_{\mathrm{r}} \times \mathrm{N}_{z}$ where the first element corresponds to $(x, z)=(1,1)$, the last to $(x, z)=(-1,-1)$ and the numbering proceeds from right to left along the horizontal layers and from top to 
Figure 1: Azimuthal velocity component $\mathfrak{u}_{\theta}$ for the Type 1 solution for $\epsilon=$ $0.083, \mathrm{Ha}=1.69 \times 10^{-3}, \mathrm{Re}=1.1 \times 10^{2}$ and $\mathrm{Fr}^{2}=4.4 \times 10^{-3}$. The profiles are shown for $z=1.0(1), 0.5(2)$ and $-0.5(3)$ in $(a)$ and for $r=1.30(1), 1.98$ (2) and $2.60(3)$ in (b).
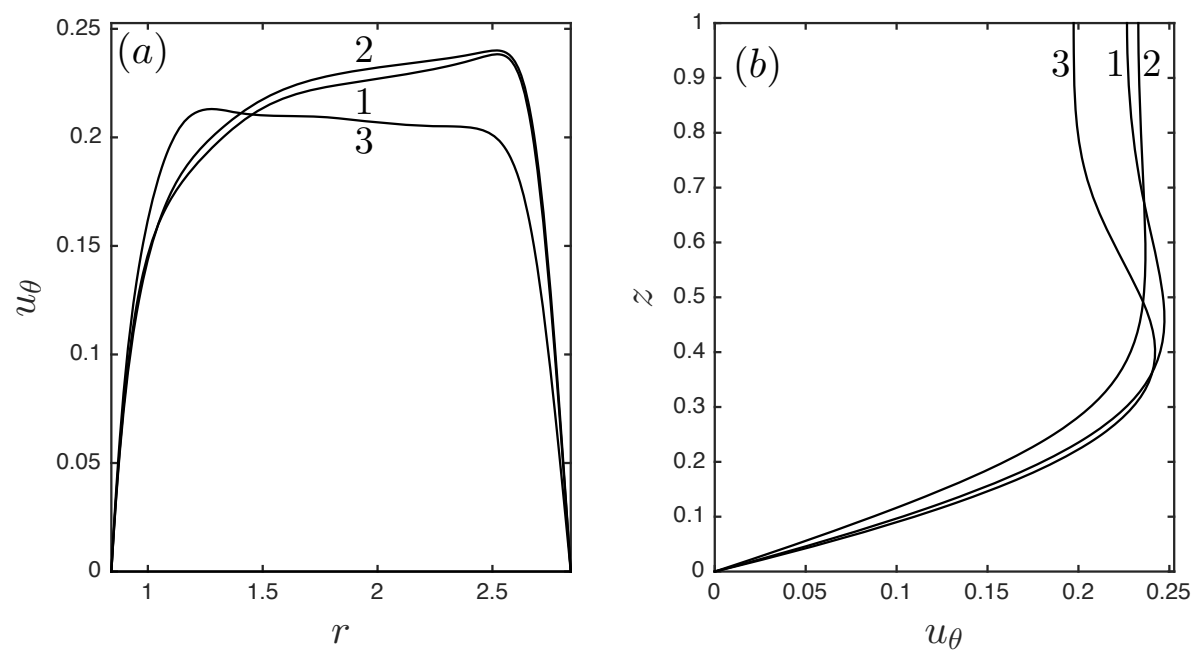

bottom vertically. Then the $\mathrm{N} \times \mathrm{N}$ differentiation and integration matrices $\hat{\mathrm{G}}_{1 x}, \hat{\mathrm{G}}_{2 x}, \hat{\mathrm{G}}_{1 z}, \hat{\mathrm{G}}_{2 z}, W_{x}$ and $W_{z}$ are obtained by the corresponding tiling of matrices $\hat{\mathrm{G}}_{1}^{\mathrm{N}_{r} \times \mathrm{N}_{\mathrm{r}}}, \hat{\mathrm{G}}_{2}^{\mathrm{N}_{r} \times \mathrm{N}_{\mathrm{r}}}, \hat{\mathrm{G}}_{1}^{\mathrm{N}_{z} \times \mathrm{N}_{z}}, \hat{\mathrm{G}}_{2}^{\mathrm{N}_{z} \times \mathrm{N}_{z}}, W^{\mathrm{N}_{\mathrm{r}} \times \mathrm{N}_{\mathrm{r}}}$ and $\mathrm{W}^{\mathrm{N}_{z} \times \mathrm{N}_{z}}$ and padding them with zeros.

\section{$3 \quad$ Numerical results}

An example of the azimuthal velocity profiles obtained by solving equations (15 ) is shown in Figure 1. We refer to this solution as Type 1 to distinguish it from another solution termed as Type 2 that will be discussed later. The profiles depend sensitively on the distance from the bottom of the layer; near the free surface the fastest flow occurs close to the outer cylinder (see lines 
Figure 2: Cross-layer three-dimensional velocity (top) and azimuthal vorticity (bottom) fields for the Type 1 solution. The right panels show close-up views of the fields near the top outer corner. The colour in the top panels represents the magnitude of the azimuthal velocity component.

$\mathbf{u}$
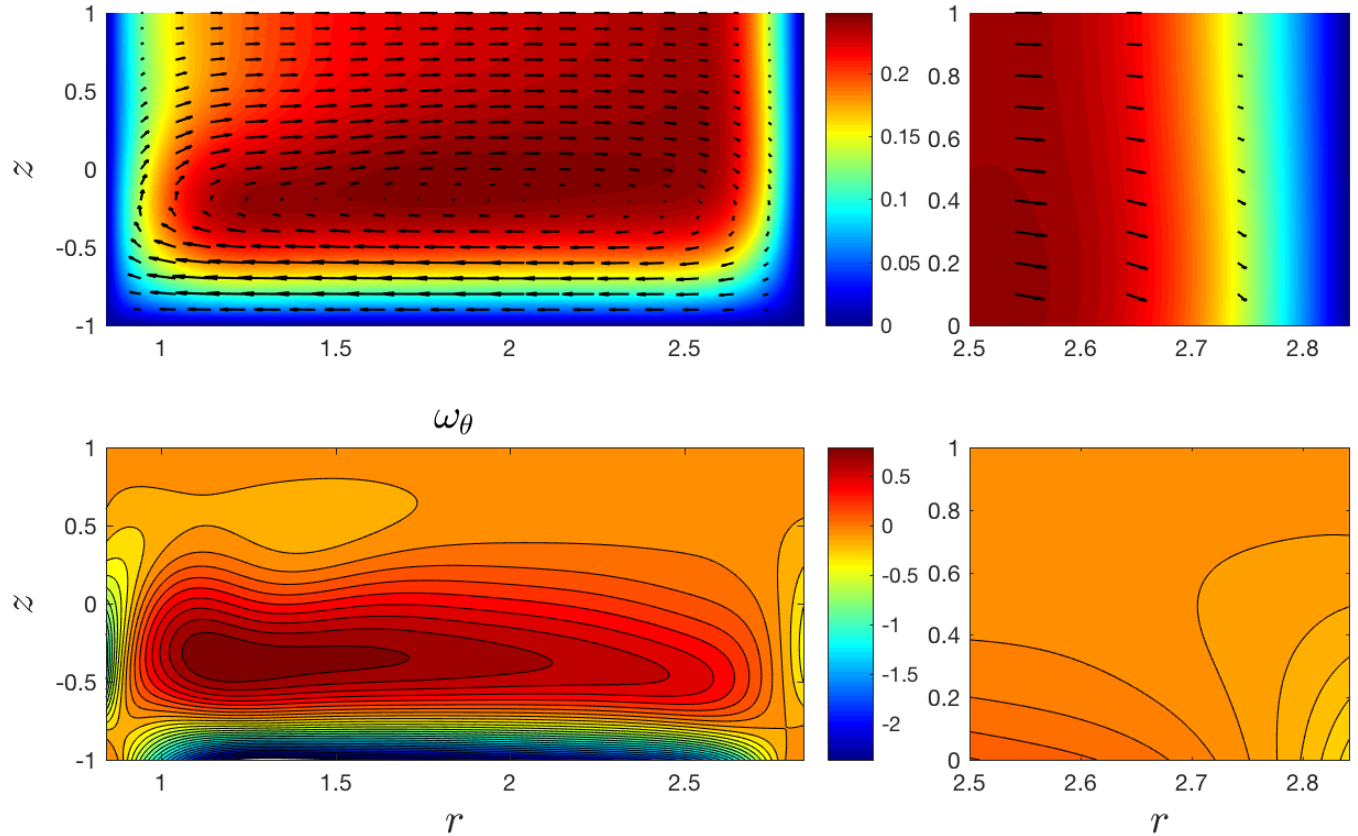

1 and 2 in Figure 1(a)), while closer to the bottom the azimuthal velocity profile becomes more of a 'plug-flow' type (line 3 in Figure 1(a)). Figure 1(b) also demonstrates that the fastest azimuthal flow occurs in the bulk of the layer rather than at the free surface.

The reason for such a counterintuitive flow structure becomes evident from Figure 2, where the nondimensional transverse flow velocity $\left(\mathfrak{u}_{r}, \in \mathfrak{u}_{z}\right)$ and azimuthal component $\omega_{\theta}$ of the vorticity vector are shown in the meridional cross-section of the layer. Even though both the maximum radial and vertical velocity components remain substantially smaller than the maximum 
Figure 3: As for Figure 2, but for the Type 2 solution.
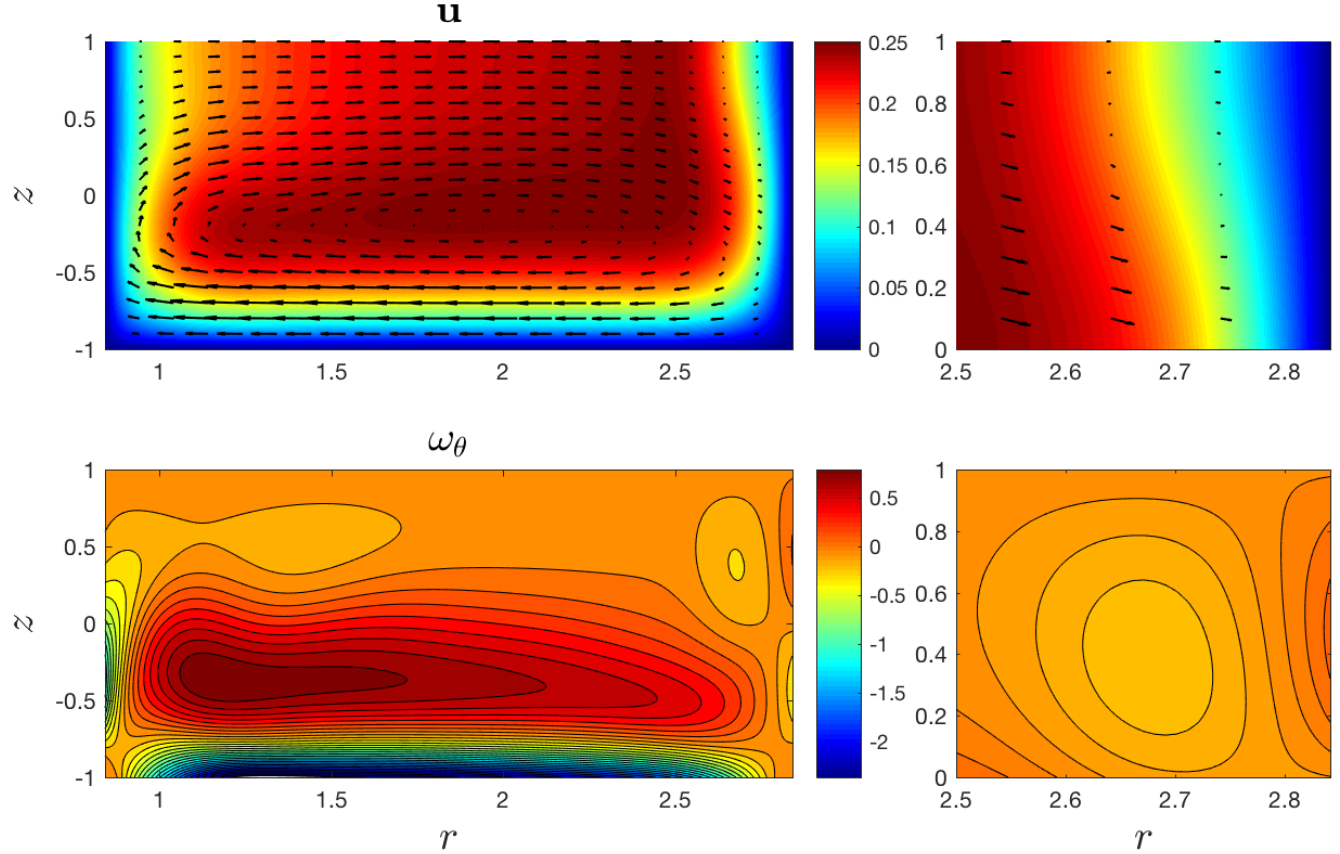

azimuthal velocity, they have a strongly pronounced effect on the overall flow. In the considered geometry fluid moves circumferentially, when observed in noninertial co-moving coordinate system as seen in the top panel in Figure 2, and experiences a centrifugal force that drives it towards the outer cylinder. However, the top-bottom symmetry is broken by the different dynamic conditions (zero tangential stress at the top and no-slip at the bottom boundaries). As a result a flow occurs in the radial direction with fluid moving outwards along the free surface, returning to the center near the bottom and rising (sinking) near the inner (outer) cylindrical boundary. The corresponding vortex is illustrated in the bottom panel in Figure 2.

We found that the convergence of iterations is very sensitive to the choice of an initial guess. One of the reasons for this is the existence of multiple 
solutions for the same set of governing parameters in some of the explored regimes. Figure 3 shows the example of a second (Type 2) solution that was found for the same set of governing parameters as those used to obtain the Type 1 solutions shown in Figures 1 and 2. As seen in the bottom panel in Figure 3 the Type 2 solution is characterised by the existence of a secondary counter-rotating vortex in the top corner near the outer cylinder. Such a vortex creates a noticeable radial counterflow along the free surface with a radial stagnation point some distance away from the outer cylinder; see the top panel in Figure 3.

Given the existence of multiple solutions a continuation procedure, where a converged steady axisymmetric solution obtained for one set of parameters is used as an initial guess for a set of slightly varied parameters, was implemented to trace the parametric evolution of the computed flow. However, such a continuation procedure was found to fail in certain regions of the parameter space. This indicates another likely feature of the problem; the existence of bifurcation points, where the type of the solution changes abruptly. Our computations also indicated that steady solutions might not exist for all parameters. At some parametric values (e.g., Re $~ 1500$ ) the iterations neither converged nor diverged with the equation residuals oscillating around some small values. Such a behaviour of steady state solvers typically occurs when steady solutions bifurcate to a time-periodic one. In conclusion, the features of numerical solutions reported above and the experimental observations of Pérez-Barrera et al. [6] serve as a strong motivation for further analytical and computational flow stability studies that will be reported in a separate dedicated publication [9].

Acknowledgements SAS acknowledges financial support from Swinburne University of Technology's Research Sabbatical Scheme under which this research has been initiated. 


\section{References}

[1] R. M. Digilov, Making a fluid rotate: circular flow of a weakly conducting fluid induced by Lorentz force. Am. J. Phys., 75(4):361-367, 2007. doi:10.1119/1.2372472 C47

[2] F.V. Dolzhanskii, V. A. Krymov and D. Y. Manin, Stability and vortex structures of quasi-two-dimensional shear flows. Sov. Phys. Usp., 33(7):495-520, 1990. doi:10.3367/UFNr.0160.199007a.0001 C47

[3] V. A. Dovzhenko, V. A. Krymov and V. M. Ponomarev, Experimental and theoretical study of a shear flow driven by an axisymmetric force. Izv. Akad. Nauk SSSR Fiz. Atm. Okeana (in Russian), 20(8):693-704, 1984. C47

[4] D. Hatziavramidis and H. C. Ku, An integral Chebyshev expansion method for boundary-value problems of O.D.E. type.

Comput. Math. Appl., 11(6):581-586, 1985.

doi:10.1016/0898-1221(85)90040-9 C50

[5] H. C. Ku and D. Hatziavramidis, Chebyshev expansion methods for the solution of the extended Graetz problem. J. Comput. Phys., 56:495-512, 1984. doi:10.1016/0021-9991(84)90109-8 C50

[6] J. Pérez-Barrera, J. E. Ortiz, E. Ramos and S. Cuevas, Instability of electrolyte flow driven by an azimuthal Lorentz force. Magnetohydrodynamics, 51(2):203-213, 2015. http://mhd.sal.1v/contents/2015/2/MG.51.2.4.R.html C47, C54

[7] S. A. Suslov and S. Paolucci, Stability of mixed-convection flow in a tall vertical channel under non-Boussinesq conditions. J. Fluid. Mech. 302:91-115, 1995. doi:10.1017/S0022112095004022 C50

[8] S. A. Suslov and S. Paolucci, Stability of natural convection flow in a tall vertical enclosure under non-Boussinesq conditions. Int. J. Heat Mass Transfer, 38:2143-2157, 1995. doi:10.1016/0017-9310(94)00348-Y C50 
[9] S. A. Suslov, J. Pérez-Barrera and S. Cuevas, Electromagnetically driven flow of electrolyte in a thin annular layer: axisymmetric solutions. J. Fluid Mech., to appear, 2017. C54

\section{Author addresses}

1. Sergey A. Suslov, Department of Mathematics, Swinburne University of Technology, Hawthorn, Victoria 3122, AUstralia mailto:ssuslov@swin.edu.au orcid:0000-0002-0998-2712

2. Sergio Cuevas, Instituto de Energias Renovables, Universidad Nacional Autónoma de México, A. P. 34, Temixco, Morelos 62580, MÉXICO 